
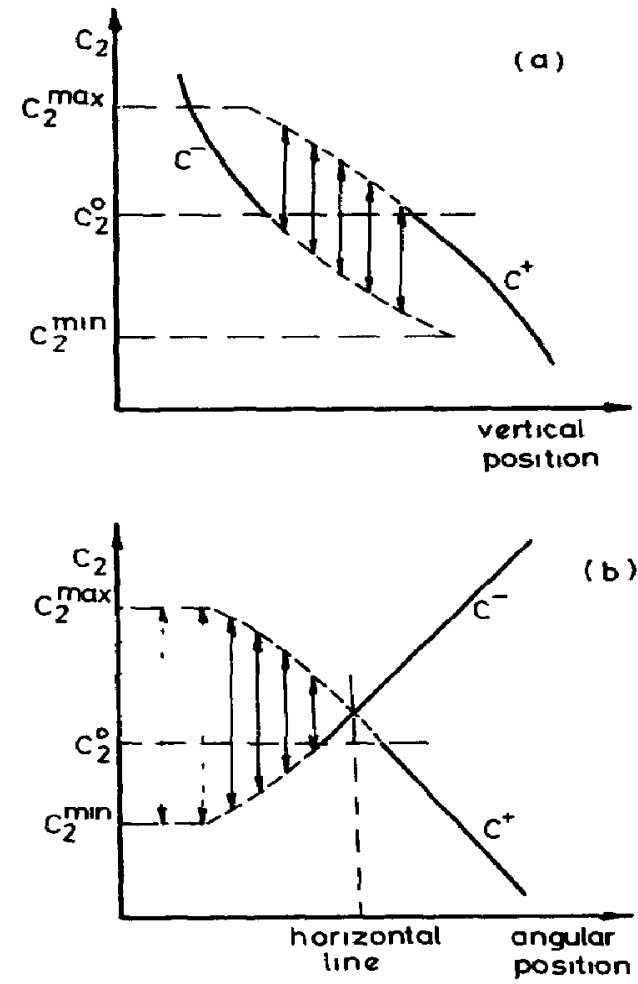

Fig 2 Dependence of the slow vanable on the position of the line $r_{2}=0$ (a), downward movement of the line, (b), rotation of the line, $\ldots$, stable states, -_, metastable states, $\uparrow$ relaxation oscillations, $\uparrow$ chaotic oscillations

state (which looks like the curves in Fig 1, with $c_{1}$ standing for volume and $c_{2}$, for pressure) and a horizontal line $c_{2}=$ const (see Fig 1c) Two states stable to infinitesimal perturbations exist at pressures between the maximum and the minimum of the $S$. shaped curve However, below a certain pressure which corresponds to the neutrally stable Maxwell construction, the gaseous state on the nght-hand branch is destabilized by growing of liquid nuclei formed due to local perturbations

Above the same pressure, expanding gaseous nuclei destabilize the hquid state on the left-hand branch Alternative states can be realized at the same pressure level only when special precautions against random nucleation help preserving the metastable superheated liquid or supercooled gas

The same picture would be observed in transitions between alternative steady states of a non-equilibrious system with a horizontal null-rate line $r_{2}=0$ Tilting it anticlockwise to the position in Fig 1(a) creates a situation when both alternative steady states are metastable On the contrary, when the null-rate line is tilted clockwise to the position in Fig I(d) so that $c_{2}{ }^{-}>c_{2}{ }^{+}$and $I\left(c_{2}{ }^{-}\right)<0, I\left(c_{2}{ }^{+}\right)>0$, the nuclei do not grow, and both steady states are stable That is the only case when hysterests can be observed in the presence of short-wave perturbations

Figure 2(a) shows how $c_{2}$ changes when the line $r_{2}=0$ inclined as in Fig 1(a) is shifted downwards When an intersection with the right-hand branch of the S-shaped curve $r_{1}=0$ first appears, It is metastable Further down, as the intersection on the left hand passes the level $c_{2}=c_{2}{ }^{0}$, where $I\left(c_{2}{ }^{0}\right)=0$, it becomes metastable as well, and chaotic oscillations anse The chaos termunates after the intersection in the right passes the level $c_{2}{ }^{\circ}$ and thus stabilizes

Figure 2(b) represents evolution of the system caused by rotating the line $r_{2}=0$ clockwise from the position in Fig 1(b) Intersections with the "stable" branches of the S-shaped curve first appear as metastable states The relaxation oscillations, instead of abruptly disappeanng, become chaotic, and their amplitude gradually decreases as the line $r_{2}=0$ comes nearer to the honzontal position At the same time, increases the wave length of perturbations needed to nucleate an alternative state (in the same manner as crystals become larger at lower supersaturation) After one of the intersections passes the level $c_{2}{ }^{0}$ with $I\left(c_{2}{ }^{0}\right)=0$, the corresponding state stabilizes and oscillations die away Anticlockwise from the horizontal position, the second intersection passes the level $c_{2}{ }^{\circ}$, and the system becomes multustable This picture demonstrates that a chaotic state appears as a natural transition from ordered relaxation oscillations to multistability

Department of Chemical Engineering Technion-Israel Institute of Technology

L M PISMEN Haifa, Israel

\section{REFERENCES}

[1] Ortoleva P and Ross J, J Chem Phys 1975633398

[2] Fife P,J Chem Phys 197644554

[3] Winfree A T, Proc 9th Symp Far Soc 1975

[4] Fife P, AMS-SIAM Symp Asymptotic Methods and Singular Perturbations, New York 1976

\title{
An evaluation of radial solid spread factors in a gas-solid packed column at trickle flow
}

\author{
(Recetved 24 May 1978, accepted 25 July 1978)
}

The trickle flow of a freely flowing solid through a packed column is a promising new gas-solnd counter-current operation with possible applications in chemical industry $[1,2]$ (e $g$ in the continuous adsorption of gases or in chemical reactions, especlally equilibrium reactions)

In previous papers [3-5] a gas solid trickle flow column was shown to behave as an almost ideal gas solid counter-current contactor (e g low pressure drop, little axial mixing and a high interphase mass transfer rate)

In the foregoing examinations the gas and solid phases were assumed to be well distributed over the column drameter Consequently the model parameters were considered to be in- dependent of the radial position in the column If, however, the local mass flux of one of the phases diverges strongly from its mean value over the cross section of the column the local capacity ratio of the phases might deviate strongly from unity thus possibly causing local equilibna between the phases This may have a pronounced effect on column performance [6]

In a gas solid tnckle flow column the solid phase is introduced through one or more feed points The particles will disperse over the packing while flowng downwards, but dependent on the spreading rate in the upper section of the column the solid phase will be maldistributed If the refiection of the column wall is also known it should be possible to calculate the solid distribution in 
any position in the column In this contribution results of the solid mass flux distributions, evaluated from solid spreading expenments the in absence of wall effects, are presented

The radial dissemination of a swarm of particles passing through a packing, in counter-current with a gas, has not been described in open literature before There are, however, two fields which are closely related to this phenomenon viz the spontaneous interparticle percolation as a mechanism for solids (de)mixing and the radial spreading of a liquid through packed column at tnckle flow conditions In both fields a diffusional model is frequently used to elucidate the mechanism of radial spreading, e g see $[7,8]$ We shall use this kind of model also as a first attempt to predict local solid mass flux distributions (possible maldistribution as defined by Groenhof for gas-liquid systems [9], is not taken into account)

The diffusional model for an axisymmetncal point source leads to the following partial differential equation (1)

$$
\frac{\partial S}{\partial h}=D\left(\frac{1}{r} \frac{\partial S}{\partial r}+\frac{\partial^{2} S}{\partial r^{2}}\right)
$$

In the absence of wall effects the following solution is obtained $[10]$

$$
\frac{S}{S_{0}}=\frac{1}{4 \pi D h} \exp \left(-\frac{r^{2}}{4 D h}\right)
$$

The solid spread factor $D$ may be obtained from the slope of a plot of $\ln \left(S / S_{0}\right)$ vs $r^{2}$ In the present investigation we determined (for the sake of convenence) the solid spread factor from the zeroth moment around the ongin of eqn (2)

$$
M_{0}=\int_{-\infty}^{+\infty} \frac{S}{S_{0}} \mathrm{~d} r=\frac{1}{\sqrt{ }(4 \pi D h)}
$$

$M_{0}$ can easily be calculated from the experimental data by numerical integration, eqn (3) then allows the evaluation of $D$

We measured $D$ injecting a freely flowing catalysts carmer (average particle size $70 * 10^{-6} \mathrm{~m}$, other properties are given in [31) from a point source (internal tube diameter $150012 \mathrm{~m}$ ) on top of the packing and collecting the particles in six coaxial annul $\left(d=24,52,86,114\right.$ and $14610^{-2} \mathrm{~m}$ ) below the gnd plate The annuli were fixed on a porous plate distributor through which air was flowing upwards The column $(015 \mathrm{~m} 1 \mathrm{~d})$ was packed with dumped PALL rings $\left(d_{p}=0015 \mathrm{~m}\right)$, cylindrical screens $\left(d_{p}=\right.$ $0010 \mathrm{~m}$ ) and RASCHIG rings The diameter of the latter packing material has been varied $\left(5,10,15\right.$ and $2010^{-3} \mathrm{~m}$ respectively) Detals of the experimental set-up have been given elsewhere [11]

Figure 1 gives a tynical example of an exnerimental distribution together wit eqn (2) Figures 2-4
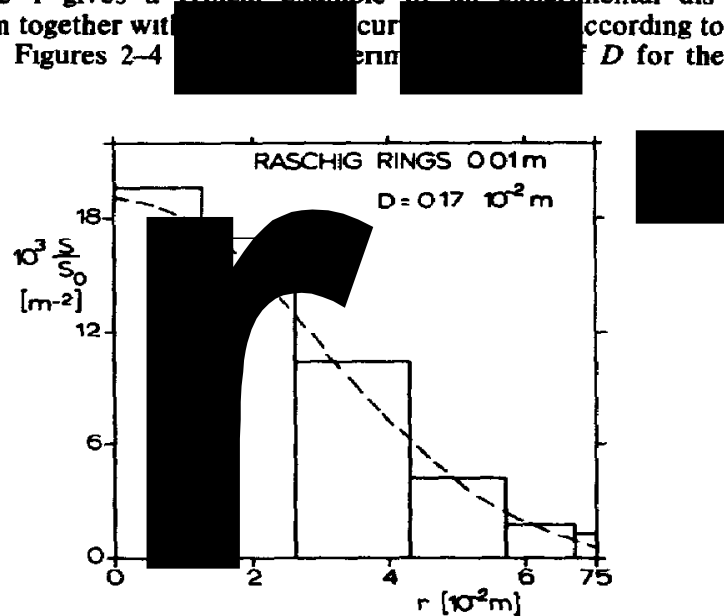

Fig 1 Experimental radial distribution RASCHIG rings $(0010 \mathrm{~m}) \Phi_{m}=104 \mathrm{~kg} / \mathrm{m}^{2} \mathrm{~s}, u_{g}=0064 \mathrm{~m} / \mathrm{s} \ldots$, calculated according to eqn (2), $D=01710^{-2} \mathrm{~m}$

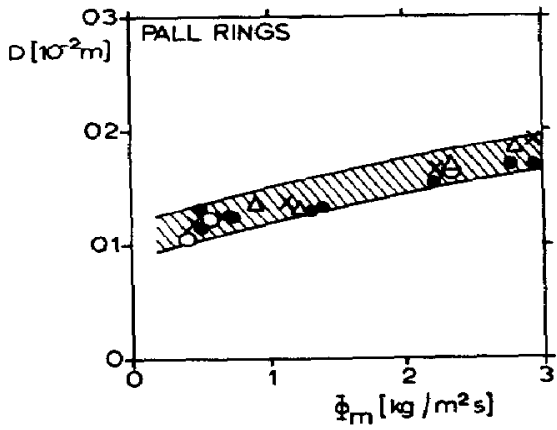

Fig 2 Solid spread factor for PALL nigs $(0015 \mathrm{~m})$ vs superficial solid mass flux

\begin{tabular}{cl}
\hline Symbol & $u_{g}[\mathrm{~m} / \mathrm{s}]$ \\
\hline$O$ & 0064 \\
$O$ & 0093 \\
$\Delta$ & 012 \\
$\times$ & 016 \\
\hline
\end{tabular}

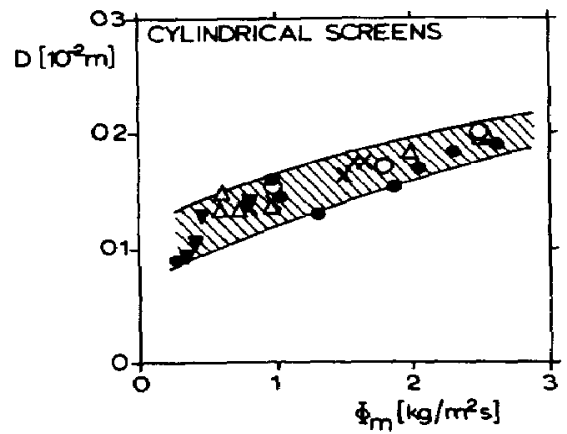

Fig 3 Sold spread factor for cylindrical screens $(0010 \mathrm{~m})$ as a function of solid mass flux (for key to symbols see Fig 2)

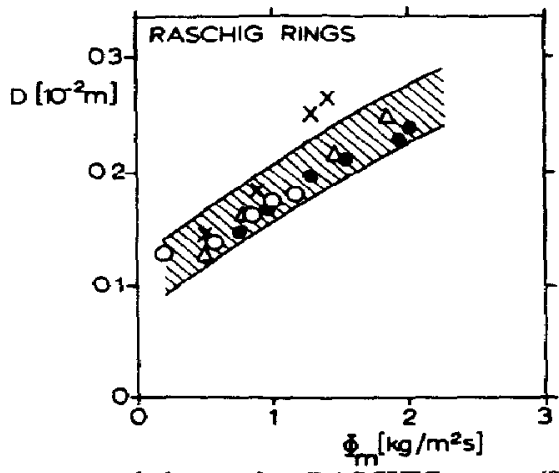

Fig 4 Solid spread factor for RASCHIG rings (0010 m) vs superficial sohd mass fiux (for key to symbols see Fig 2)

difterent packings as a function of the superficial solid mass flux at different gas flow rates In the investigated region the solid spread factor is independent of the superficial gas velocity and increases with an increased solid mass fiux In gas-liquid systems the opposite has been observed, the liquid spread factor is independent of the liquid velocity[12] and increases with an increased gas velocity [13]

In gas-liquid systems the packing size was found to have a dominant effect on the hquid spread factor $[12,14,15]$ In Fig 5 the radial solud spread factor for RASCHIG nings is plotted vs 


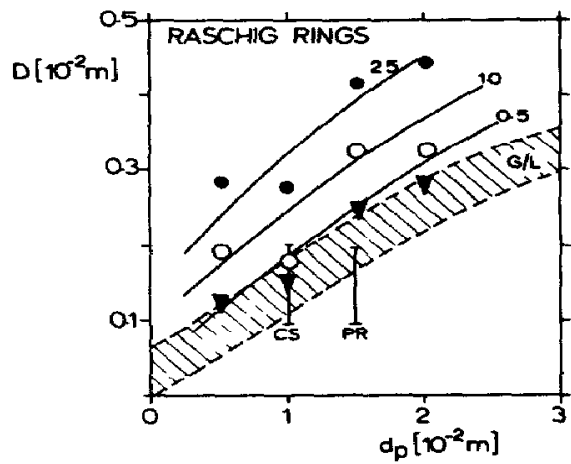

Fig 5 Sold spread factor for RASCHIG nngs vs packing diameter

\begin{tabular}{|c|c|c|}
\hline Symbol & $\Phi_{m}\left(\mathrm{~kg} / \mathrm{m}^{2} \mathrm{~s}\right)$ & $\begin{array}{c}\text { (CS cylindncal screens } \\
\text { PR PALL nngs) }\end{array}$ \\
\hline $\begin{array}{l}7 \\
0\end{array}$ & $\begin{array}{l}05 \\
10 \\
25\end{array}$ & \\
\hline
\end{tabular}

the packing diameter The ranges of the expenmental data for PALL rings and cylindrical screens are also given Data for gas liquid systems lie in the shaded area $[12,14,15]$ Analogous to the hquid spread factor the solid spread factor increases at enlarged packing sizes At low superficial solid mass fuxes the expenmental data for RASCHIG rings almost coincide with the data for gas liquid systems, if the solid mass flux increases the values for the solid spreád factor are considerably larger The expenmental values for cylindncal screens and PALL rings, however, are much closer to those for gas liquid systems

Acknowledgements - The authors are much indebted to DSM for the financial support (for A W M R) during the course of this work and to $F$ J $M$ Eijsink and $H$ H Nijkamp who carned out the experiments

†Present address Koninklike/Shell-Laboratorium, Am-
sterdam

Department of Chemical Engineerng Twente Unversity of Technology PO Box 217

Enschede, The Netherlands

\section{NOTATION}

$D$ solid spread factor, $\mathrm{m}$

$d_{p}$ nominal packing size, $m$

$h$ distance between injection point and packing supporting gnd, $m$

$M_{0}$ zeroth moment around the $7 g i n$ of eqn (2), $\mathrm{m}^{-1}$

$r$ distance from column axis, $m$

$S$ local solid mass flux, $\mathrm{kg} \mathrm{m}^{-2} \mathrm{~s}^{-1}$

$S_{\mathrm{o}}$ solid mass flow from point source, $\mathrm{kg} \mathrm{s}^{-1}$

$u_{\mathrm{z}}$ superficial gas velocity, $\mathrm{m} \mathrm{s}^{-1}$

$\Phi_{m}$ superficial solıd mass flux, $\mathrm{kg} \mathrm{m}^{-2} \mathrm{~s}^{-1}$

\section{REFERENCES}

[1] Claus G, Vergnes F and Le Goff P, Can J Chem Engng 197654143

[2] Van Swaaij W P M, Proc Int Symp Chem Reac Engng (Houston 1978), Am Chem Soc Symp Ser part II

[3] Roes A W $M$ and Van Swaai] W P M, Chem Engng $J$, to be published

[4] Roes A W M and Van Swaaly W P M, Chem Engng $J$, to be published

[5] Roes A W M and Van Swaal] W P M, Chem Engng J, to be published

[6] Groenhof H C, Ph D Thesis, Groningen 1972

[7] Bndgewater J, Sharpe $\mathbf{N} \mathbf{W}$ and Stocker D C, Trans Instn Chem Engrs 196947 T114

[8] Chla Z and Schmidt O, Coll Czech Chem Comm 195722 896

[9] Groenhof H C, Chem Engng J 197714193

[10] Carslaw $\mathrm{H} S$ and Jaeger J C, Conduction of Heat in Solids 2nd Edn, p 50 Oxford at the Clarendon Press, London 1959

[11] Roes A W M, Ph D Thesis, Enschede 1978

[12] Bemer G G and Zuiderweg F J, Chem Engng Sci 197833 1637

[13] Dutkal E and Ruckenstein E, Chem Engng Sct 197025 483

[14] Brignole E A, Zacharonek $G$ and Magnosio J, Chem Engng Sct 1973281225

[15] Onda $\mathbf{K}$, Takeuchı $\mathbf{H}$, Maeda $Y$ and Takeuchı $N$, Chem Engng Scr 1973271677

\title{
Design of a pulse diffusivity apparatus for improved sensitivity
}

\author{
(Received 7 Apnl 1978)
}

Several techniques have been proposed in the literature to measure effective diffusivities in porous catalysts The counterdiffusion cell technique[1], which applies basic diffusion theory, is a steady-state, single pellet measurement This technique was used in our laboratory for measuring diffusivitues for a variety of catalyst supports [2] However, single pellet techniques pose sampling problems if a non-uniform collection of pellets is to be characterized

The pulse (or chromatographic) technique examines the eluted concentration pulse from a packed column after a plug of tracer is injected into the carner gas stream Waldram [3] reviewed diffusivity measuring techmiques and concluded that pulse techniques often falled to provide a sensitive measure of the effective diffusivity because the overall dispersion in a packed bed was only a weak function of the intraparticle diffusivity Scott et al [4] observed that the use of a pellet string reactor enables one to reduce axial dispersion effects and thus to enhance the contribution from intrapellet diffusion to the overall pulse dispersion This phenomenon was recently confirmed by Hsiang and Haynes [5] Haynes [6] also theoretically analyzed the effects of carner gas flow rate and catalyst pellet diameter on the sens1tivity of the diffusivity measurement in a bidispersed catalyst support

In practical situations, the catalyst pellet diameter is usually limited to a narrow range Also, the highly sensitive flow-through type detectors which are used for diffusivity studies are often 